\title{
La gestión directiva y los rendimientos de alumnos en educación media superior
}

School management and student performance in upper secondary education

Gestão diretiva e desempenho dos alunos no ensino médio

José Ángel Vera Noriega

Universidad de Sonora, México.

Jose.vera@unison.mx

https://orcid.org/0000-0003-2764-4431

Luis Enrique Fierros Dávila

Universidad de Sonora, México

luisenrique.fierros@unison.mx

https://orcid.org/0000-0002-2212-3445

José Alberto Fraijo Figueroa

Universidad de Sonora, México

jose.fraijo@unison.mx

https://orcid.org/0000-0001-9365-9282 


\section{Resumen}

En la actualidad, el sistema educativo en México afronta ciertas situaciones de cambio y reestructuración que afectan el funcionamiento de todos los niveles educativos. De hecho, algunas investigaciones han identificado diferentes elementos que impiden el desarrollo normal del proceso educativo, como el desempeño del docente en clase, el interés y motivación del alumno, el ambiente donde se desenvuelve la institución, y un aspecto de gran relevancia: el papel del director en su institución. Por eso, el objetivo del estudio fue estimar la relación entre las características del director del plantel como variable de contexto y su relación con el rendimiento de los alumnos. Para ello, participaron 265 directores de instituciones de educación media superior del estado de Sonora, elegidos aleatoriamente, a quienes se les aplicó un instrumento que evaluaba elementos de sus contextos. Se realizó un análisis multivariado discriminante que permitió diferenciar a los directores de estudiantes con alto y bajo rendimiento, con una efectividad de pronóstico de $88.40 \%$. Las variables que más contribuyeron a las diferencias fueron juntas y cursos, infraestructura y salario y problemas. Se puede concluir que solamente las acciones que están en el radio de acción de los directores son las que efectivamente coadyuvan a la generación de estudiantes con alto rendimiento.

Palabras clave: director de escuela, educación media superior, rendimiento académico.

\section{Abstract}

Today the educational system in Mexico faces certain situations of change and restructuring that affect the functioning of all educational levels. Some researches have identified different factors that affect the educational process, such as the teacher's performance in class, the student's interest and motivation, the environment where the institution develops, and an aspect of great relevance: the role of the principal in his or her institution. The objective of the study was to estimate the relationship between the characteristics of the school principal as a context variable and its relationship with the performance of students at the school, evaluated with a measure of mastery based on the school curriculum. A total of 265 randomly selected high school principals from the state of Sonora participated in the study, who were administered an instrument that evaluates elements of the context in which they work. A multivariate discriminant analysis was carried out to differentiate principals of students with 
high and low performance, with a predictive effectiveness of $88.40 \%$; the variables that most contributed to the differences were: Meetings and Courses, Infrastructure, and Salary and Problems. It is observed that only the actions that are within the principals' radius of action are the ones that effectively contribute to the generation of the most effective results.

Keywords: school principal, upper secondary education, academic performance.

\section{Resumo}

Atualmente, o sistema educacional no México enfrenta certas situações de mudança e reestruturação que afetam o funcionamento de todos os níveis educacionais. De fato, algumas investigações identificaram diferentes elementos que impedem o normal desenvolvimento do processo educacional, como o desempenho do professor em sala de aula, o interesse e a motivação do aluno, o ambiente em que a instituição atua e um aspecto de grande relevância. : a função do diretor em sua instituição. Portanto, o objetivo do estudo foi estimar a relação entre as características do diretor do campus como variável de contexto e sua relação com o desempenho dos alunos. Para tanto, participaram 265 diretores de instituições de ensino médio do estado de Sonora, escolhidos aleatoriamente, aos quais foi aplicado um instrumento que avaliou elementos de seus contextos. Foi realizada uma análise multivariada discriminante que permitiu diferenciar os diretores dos alunos com alto e baixo desempenho, com eficácia prognóstica de $88,40 \%$. As variáveis que mais contribuíram para as diferenças foram diretorias e cursos, infraestrutura e salário e problemas. Pode-se concluir que apenas as ações que estão no raio de ação dos diretores são aquelas que efetivamente contribuem para a geração de alunos com alto desempenho.

Palavras-chave: diretor de escola, ensino médio, desempenho acadêmico. Fecha Recepción: Enero 2021 Fecha Aceptación: Julio 2021 


\section{Introducción}

En la actualidad, el sistema educativo en México afronta ciertas situaciones de cambio y reestructuración que afectan el funcionamiento de todos los niveles educativos (Organización para la Cooperación y el Desarrollo Económicos [OCDE], 2020). Un ejemplo de ello es la propuesta de reforma del nuevo modelo educativo 2019, donde se plantea una coordinación entre todos los niveles de educación, incluido el de educación media superior (EMS) en el programa básico obligatorio con el propósito de brindar a los ciudadanos calidad en su formación educativa (Secretaría de Educación Pública [SEP], 2019). Para eso, sin embargo, "se debe contar con una clara y eficiente gestión escolar al interior de los establecimientos educacionales" (López, 2010, p. 148).

En tal sentido, la EMS tiene algunos retos que se localizan en los rubros relacionados con a) acceso, equidad y cobertura; b) calidad y c) gestión, integración y coordinación del sistema (Uribe, López-Córdova, Mancera y Barrios, 2012). Los primeros dos incisos fueron planteados nuevamente por Bustamante, Székely y Martínez (2017), quienes destacan lo crucial de ser atendidos para optimizar el funcionamiento de la EMS. Para ello, se debe hacer énfasis en el uso de los recursos y en cómo son aprovechados para conseguir los objetivos del plantel en materia de calidad educativa (Pacheco, Robles y Ospino, 2018).

No obstante, es en la tarea de articular los objetivos del sistema educativo y las metas institucionales planteadas para el logro de la calidad educativa donde reside, en ocasiones, una ruptura entre los deberes asignados y la implementación de las acciones propuestas por el sistema (Quintana Torres, 2018). En este marco, la gestión escolar surge como una herramienta de trabajo conjunto que debe permitir tomar postura en lo que al logro de metas se refiere. Esto, por supuesto, demanda un trabajo conjunto para ofrecer acciones que se ajusten a las condiciones de cada institución.

\section{Los directores y su relación con el rendimiento de los alumnos}

El director es la persona que tiene la función de tomar decisiones para la administración del recurso financiero y del personal, así como para atender necesidades relacionadas con el desarrollo del conocimiento académico. Esta visión comprende una capacidad de gestión y entendimiento de las dinámicas de los elementos que integran todo el 
sistema educativo del plantel (Acosta, 2009), aunque se sabe que en algunos casos los directivos carecen de capacidades para solucionar las demandas (Sardon, 2017).

En este sentido, diferentes estudios sobre la administración de organizaciones educativas señalan una alta correlación entre la calidad de la enseñanza, el aprendizaje que tiene lugar en las escuelas y la calidad del liderazgo del director (Acosta, 2009; Guzmán, 2018; Horbath y García, 2014; Pacheco et al., 2018).

Peniche y Cisneros-Cohernourm (2013) explican que las funciones del director también incluyen acciones que se orientan a la mejora del liderazgo instruccional y del desarrollo personal y profesional de sus docentes. Sin embargo, para plantear propuestas efectivas que permitan optimizar la dinámica educativa se deben concretar trabajos de investigación empíricos (Tolozano, Ferrer y Forgas, 2017).

Todo lo anterior nos lleva a considerar las funciones que el director realiza o debería realizar como elemento importante en el contexto donde se desarrollan los estudiantes, pues sus decisiones pueden impactar en el rendimiento escolar de los alumnos (Bitar, 2012; Vélez, 2012).

Sin embargo, de manera general (y con base en la bibliográfica disponible) se puede afirmar que son escasas las investigaciones que relacionen la gestión directiva con el rendimiento académico de los alumnos, pues la mayoría de las veces el liderazgo se mide en relación con actitudes y opiniones de docentes o alumnos (tabla 1). 
Revista Iberoamericana

de las Ciencias Sociales y

Humanísticas

ISSN: $2395-7972$

Tabla 1. Variables estudiadas en la relación ejercicio del director y efecto en el rendimiento escolar de los estudiantes

\begin{tabular}{|c|c|}
\hline Autor (año) & Variables \\
\hline $\begin{array}{l}\text { Chain } \quad(1995) \quad y \\
\text { Pérez-Franco }(2001)\end{array}$ & $\begin{array}{l}\text { Factores sociales y económicos referidos a condiciones } \\
\text { materiales de vida y condiciones culturales. }\end{array}$ \\
\hline Elmore (2004) & $\begin{array}{l}\text { Práctica y competencia pedagógica. } \\
\text { Promueve el aprendizaje continuo del personal, se genera una } \\
\text { condición de aprender y mejorar más que cumplir con dictados } \\
\text { institucionales. } \\
\text { Ejercicio recíproco de responsabilidades y capacidades. }\end{array}$ \\
\hline Garay y Uribe (2006) & $\begin{array}{l}\text { Focalización en los aprendizajes en un ambiente ordenado (con } \\
\text { énfasis en la disciplina de los procesos pedagógicos) en las } \\
\text { distintas formas de aprendizaje y ritmo de los alumnos. } \\
\text { Convicción en creer sin limitaciones (altas expectativas) en las } \\
\text { capacidades de aprendizaje de los estudiantes. }\end{array}$ \\
\hline Hunt (2009) & $\begin{array}{l}\text { Efectividad docente y relación con el clima de la escuela. } \\
\text { El liderazgo y supervisión ejercidos por el director. }\end{array}$ \\
\hline $\begin{array}{l}\text { CEPPE (2009) y } \\
\text { Bush y Glover (2003, } \\
\text { citados por Vázquez, } \\
\text { 2013) }\end{array}$ & Liderazgo instruccional (o liderazgo educativo). \\
\hline Vélez (2012) & $\begin{array}{l}\text { Gestión del director sobre parámetros técnico-administrativo- } \\
\text { docentes y mejoría en calidad educativa. }\end{array}$ \\
\hline $\begin{array}{l}\text { Tolozano et al. } \\
(2017)\end{array}$ & $\begin{array}{l}\text { Orienta y crea las condiciones en el uso de los diferentes } \\
\text { recursos. } \\
\text { Funciones del director: planeación, organización, mando, } \\
\text { ejecución, control y evaluación de todo el proceso. }\end{array}$ \\
\hline Sardon (2017) & $\begin{array}{l}\text { Prácticas del liderazgo transformacional: } \\
\text {-La motivación inspiradora. } \\
\text {-La estimulación intelectual. } \\
\text {-La influencia idealizada. } \\
\text {-La consideración individualizada. }\end{array}$ \\
\hline Pacheco et al. (2018) & $\begin{array}{l}\text { Capacidad de gestión. } \\
\text { Toma de decisiones. } \\
\text { Capacidad de coordinación de acciones y su implementación. }\end{array}$ \\
\hline
\end{tabular}

Fuente: Elaboración propia

En los anteriores estudios se enfatiza que el liderazgo del director es una de las variables que influye en la eficacia, pero no se explica cómo y en qué medida. De hecho, las diferentes investigaciones concluyen que a) los efectos del liderazgo (directos e indirectos) explican $25 \%$ de los efectos totales en los logros escolares (Vázquez, 2013); b) el liderazgo escolar es la segunda variable más importante en el aprendizaje de los alumnos (la primera es la enseñanza del docente en el salón de clase) (Bravo y Verdugo, 2007); c) los líderes 


\section{Revista Iberoamericana \\ de las Ciencias Sociales y Humanísticas}

ISSN: $2395-7972$

escolares (directores) mejoran la enseñanza y el aprendizaje a través de su influencia en la motivación del personal, en el compromiso y en las condiciones de trabajo; y d) el liderazgo escolar tiene mayor influencia en la escuela y en los alumnos cuando es ampliamente distribuido.

Por su parte, Maureira (2006) plantea que la investigación en esta área ha identificado algunas características comunes asociadas al liderazgo exitoso: a) fuerza en los propósitos, que se refiere a la capacidad para imprimir dinamismo o a la mediación entre los agentes de cambio negativos; b) involucrar al cuerpo académico en la toma de decisiones, esto en relación con la capacidad del director para compartir el liderazgo con su equipo directivo e integrar a los maestros en ciertos procesos de toma de decisiones; y c) competencia profesional en los procesos de enseñanza y aprendizaje, característica esencial para establecer una ascendencia sobre los maestros.

Asimismo, Sardon (2017) encuentra una alta relación significativa entre las variables de liderazgo transformacional y gestión escolar. De acuerdo con el autor, al ejercer su liderazgo, el directivo debe saber comunicarse con las personas del plantel escolar y actuar según la situación lo demande en cumplimiento con los objetivos establecidos.

Por todo lo anterior, el objetivo de la presente investigación fue estimar la relación entre las características del director del plantel como variable de contexto y su relación con el rendimiento de los alumnos de los planteles. La hipótesis general del estudio se asume como una diferenciación entre los niveles de rendimiento y los estilos de gestión directiva.

\section{Metodología}

\section{Población}

De los 651 directores en funciones, se eligieron de manera aleatoria 272. Sin embargo, después de revisar cuidadosamente la base de datos, se eliminaron siete participantes porque presentaban información incompleta, por lo que la muestra quedó conformada por 265 sujetos. Para esto, se usó la tabla elaborada por Tagliacarne (1968) para poblaciones infinitas, con $5 \%$ de error para una probabilidad de 50 sobre 50 y un intervalo de confianza de $95 \%$ equivalente a dos sigmas (Sierra-Bravo, 1985). La muestra total fue ponderada de la siguiente manera: $40.66 \%$ para el segundo semestre, $31.51 \%$ para el cuarto semestre y $27.78 \%$ para 
Revista Iberoamericana

de las Ciencias Sociales y

Humanísticas

ISSN: 2395 - 7972

el sexto semestre. Todas las instituciones de EMS y la muestra estuvieron diseñadas para el estado de Sonora, México.

\section{Instrumento}

Se diseñó un cuestionario compuesto por 40 reactivos en varias dimensiones para evaluar diversos elementos del contexto donde se desenvuelven los directores que participaron en el estudio. A continuación se describen esas dimensiones:

- Datos generales: Estuvo compuesta por 11 reactivos: sexo, estado civil, cantidad de hijos, tipo y nombre de la escuela, antigüedad en la docencia, antigüedad en la institución, grado académico, área de estudios, actividades laborales externas a la institución, productividad y participación en la carrera magisterial.

- Trabajo directivo: Estuvo compuesta por lo siguiente: reuniones con padres de familia, juntas de planeación con profesores, juntas con supervisores y autoridades de la Secretaría de Educación y Cultura (SEC) o de la propia institución, cursos de capacitación para maestros, reuniones con alumnos, gestiones para la adquisición de nuevo equipo, reuniones para elaboración de proyectos de investigación, actividades culturales, deportivas o sociales con alumnos y promoción de eventos académicos externos entre profesores del plantel.

- Condiciones de trabajo y clima organizacional: Esta procuró recabar información sobre la evaluación que el director hace de su entorno laboral y su relación con su práctica, así como sus niveles de satisfacción. Las subescalas incluidas fueron atención a la organización académica, equipamiento, aprovechamiento del alumno, asistencia y puntualidad, infraestructura, salario, desempeño y comunicación. Los reactivos que constituyeron este apartado fueron atención al personal de biblioteca, apoyo al trabajo conjunto de profesores y alumnos, realización de eventos académicos, equipamiento de aulas, laboratorios y talleres, sobre su habilidad para coordinar equipos de trabajo, condiciones laborales en su institución, comunicación con alumnos, profesores y padres de familia, etc.

- Gestión y actividades directivas: El objetivo fue obtener información sobre la actividad sustantiva del director, la cual quedó conformada por cuatro subescalas: gestión administrativa, planeación, gestión docente y relación con padres de familia. Los reactivos se enfocaron en gestión de recursos financieros para mejorar la 
Revista Iberoamericana

de las Ciencias Sociales y

Humanísticas

ISSN: 2395 - 7972

infraestructura, actividades extracurriculares, estudios de posgrado para sus profesores, incentivar a los profesores a continuar sus estudios, informar a los padres sobre el desempeño de sus hijos, etc.

- El alumnado: Esta dimensión estuvo compuesta por cuatro subescalas: mejora del rendimiento de los alumnos, motivación y capacitación, problemas, y más problemas. Los reactivos se centraron en la comunicación con los alumnos, comunicación entre alumnos y profesores, problemas administrativos, innovación de las tecnologías de la información y de la comunicación, promoción de becas, etc.

\section{Procedimiento}

- Primera etapa: En esta se estableció el lugar donde se desarrollaría el proceso y la recepción de los formatos de logística.

- Segunda etapa: El monitor se encargó de gestionar la aplicación de los exámenes en la institución. Se identificó y acreditó el lugar de procedencia. Se indicó al director del plantel el objetivo y las dinámicas que el equipo desarrollaría en la escuela. Asimismo, se solicitó el apoyo para realizar de las entrevistas a los directores participantes. Cuando estos contestaron el instrumento, se revisó para verificar que no hubiese faltado algún reactivo por contestar. En dichos casos, se solicitó que los terminaran (si era posible). No hubo límite de tiempo. Todos los directores firmaron consentimiento informado.

\section{Resultados}

\section{Análisis de conglomerados del rendimiento académico}

Antes de enfocarnos en el objetivo general de este trabajo, primero se debe integrar y diferenciar a los conglomerados con base en las variables de logro de los estudiantes. Para esto, se utilizó el análisis de conglomerados, el cual fue del tipo no jerárquico mediante el procedimiento de K-medias. Se introdujo como variable el promedio global que los estudiantes obtuvieron de forma individual en la aplicación del instrumento de logro, que incluye matemáticas, español y ciencias. Los detalles sobre la composición y distribución de medidas de tendencia central y dispersión de los puntajes globales de logro se encuentran referidos en Vera, Fierros y Peña (2014) y Fierros (2014). En las referencias anteriores se 


\section{Revista Iberoamericana de las Ciencias Sociales y Humanísticas}

exploraron modelos de dos, tres y cuatro conglomerados para abarcar el mayor número de sujetos y de valores por sujeto (valores altos, medios y bajos). En definitiva, el último fue el que cumplía con un número de iteraciones menor a 15, y las desviaciones de los centroides nunca fueron mayores a dos desviaciones estándar para cada sujeto. Así, se pudieron identificar el grupo de mejor rendimiento (conglomerado 4), dos grupos de rendimiento medio (conglomerados 3 y 2) y un grupo de bajo rendimiento (conglomerado 1).

Como puede observarse en la tabla 2 , de acuerdo con los robustos valores $F$ de la Anova, se puede señalar que el promedio global fue lo suficientemente consistente como para explicar, sin lugar a duda, la formación de los conglomerados que se reportan. El método de las K-medias permite asignar a cada observación el clúster (conglomerado) que se encuentra más próximo en términos del centroide (media o distancia). En este sentido, puede observarse, en la misma tabla, que los centroides están muy bien definidos y que describen sólidamente la constitución de los cuatro conglomerados, considerando además el robusto valor de F. Asimismo, el número de alumnos considerados, el número de iteraciones, el valor de los centroides y los robustos valores de F nos permite considerar con confianza los análisis realizados y resultados obtenidos hasta el momento.

Tabla 2. Conglomerados y parámetros de la solución obtenida

\begin{tabular}{|c|c|c|c|c|c|c|c|c|}
\hline $\begin{array}{c}\text { Conglomerad } \\
\text { os }\end{array}$ & \multicolumn{9}{|c|}{} \\
\hline & $\begin{array}{c}\text { COBAC } \\
\mathrm{H}\end{array}$ & $\begin{array}{l}\text { Alu } \\
\mathrm{m}\end{array}$ & $\begin{array}{c}\text { CONALE } \\
\mathrm{P}\end{array}$ & $\begin{array}{l}\text { Alu } \\
\mathrm{m}\end{array}$ & $\begin{array}{l}\text { DGET } \\
\text { I }\end{array}$ & $\begin{array}{l}\text { Alu } \\
\mathrm{m}\end{array}$ & $\begin{array}{c}\text { Privada } \\
\text { s }\end{array}$ & $\begin{array}{l}\text { Alu } \\
\mathrm{m}\end{array}$ \\
\hline 1 & 33.50 & 22 & 26.98 & 25 & 27.16 & 122 & 34.00 & 61 \\
\hline 2 & 41.15 & & 38.33 & & 34.72 & & 45.21 & \\
\hline 3 & 49.72 & & & & 42.45 & & 52.94 & \\
\hline 4 & 61.10 & 39 & 45.90 & 21 & 50.04 & 22 & 63.51 & 23 \\
\hline F & 794.69 & & 352.46 & & 1223 & & 808.57 & \\
\hline Sig. & 0.000 & & 0.000 & & 0.000 & & 0.000 & \\
\hline Iteraciones & 12 & & 13 & & 12 & & 12 & \\
\hline Distancia & 7.647 & & 7.609 & & 7.565 & & 7.731 & \\
\hline Alumnos & 168 & & 69 & & 452 & & 138 & \\
\hline
\end{tabular}

Fuente: Elaboración propia

En el caso de los alumnos del Colegio de Bachilleres del Estado de Sonora $(\mathrm{COBACH})$, puede observarse una diferencia significativa entre los conglomerados de alto y bajo rendimiento (medias de 61.10 y de 33.5, respectivamente). Una diferencia similar se 


\section{Revista Iberoamericana de las Ciencias Sociales y Humanísticas}

aprecia en los grupos que corresponden a Colegio Nacional de Educación Profesional Técnica (CONALEP) (45.9 y 26.98 de medias para los grupos de alto y bajo rendimiento, respectivamente). En lo que respecta a los alumnos de Dirección General Tecnológica Industrial (DGETI), el grupo de alto rendimiento muestra una media de 50.04, y el de bajo rendimiento 27.16. Finalmente, los alumnos de alto rendimiento de las preparatorias privadas presentan una media de 63.51, mientras que los de bajo rendimiento tienen 34. En estos términos, puede señalarse que el mejor desempeño corresponde al grupo de alumnos de las preparatorias privadas.

Al respecto, cabe señalar que los planteles que aportan un mayor número de alumnos de alto rendimiento se encuentran ubicados en centros poblacionales con infraestructura y condiciones materiales adecuadas (p. ej., Hermosillo, Ciudad Obregón, Magdalena, etc.). Estos planteles tradicionalmente han sido reconocidos por la alta calidad de sus egresados y porque desarrollan sus actividades en condiciones favorables (De la Cruz, 2016). De hecho, la mayoría de estos alumnos solamente tienen como obligación principal su formación académica. Sin embargo, no se debe obviar que incluso en estas localidades también se halla presente el fenómeno del bajo rendimiento, lo que permite inferir que existen otras variables que influyen en la persistencia de dicho problema.

Ahora bien, quienes presentan un mayor número de alumnos con bajo rendimiento son los planteles que se encuentran en poblaciones desfavorecidas (p. ej., Pueblo Yaqui, Nacozari, Navojoa, Huatabampo, etc.), las cuales suelen tener los más altos índices de pobreza del estado.

Aunado a lo anterior, cabe resaltar que la asignación del turno para cursar estudios depende de las calificaciones de cada alumno, es decir, a los de mejor rendimiento se les asignan las clases matutinas, mientras que el turno vespertino queda disponible para los demás estudiantes. Sobre esta división de horarios, Vera, Huesca y Laborín (2011) comentan lo siguiente:

El turno vespertino y el consumo de bebidas alcohólicas se relacionan con la pertenencia al grupo de bajo rendimiento escolar. Los de alto rendimiento presentan relación directa con el patrimonio cultural, su promedio actual y la discusión de lectura previa; sin embargo, las técnicas pedagógicas implementadas por parte del docente, así como las utilizadas por parte de los alumnos no garantizan la adhesión al grupo de alto rendimiento (p. 62). 
Revista Iberoamericana

de las Ciencias Sociales y

Humanísticas

ISSN: $2395-7972$

Por otra parte, se observa que el segundo semestre aporta mayor número de alumnos de alto rendimiento, mientras que lo contrario sucede en el sexto semestre. Este efecto es explicado por Vera et al. (2011), quienes señalan que en primero y segundo semestre no es posible observar cambios importantes en los niveles de rendimiento asociados a las variables de contexto. Sin embargo, a partir del cuarto las diferencias comienzan a hacerse importantes y las variables de contexto comienzan a desarrollar un papel fundamental en la probabilidad de terminar con éxito los estudios de bachillerato.

\section{Descriptivos de las dimensiones de gestión directiva}

La primera subescala (denominada Trabajo directivo) estuvo constituida por dos factores: actividades y reuniones (4 reactivos) $($ media $=2.61$; desviación estándar $=0.91)$, y juntas y reuniones $(5$ reactivos) (media $=2.36$; desviación estándar $=0.87)$. En conjunto, los valores fueron los siguientes: KMO de 0.831, varianza explicada de 49.01 y alfa de Cronbach de 0.784 .

De la subescala denominada Condiciones de trabajo y clima organizacional se extrajeron seis factores: atención a la organización académica (4 reactivos) (media = 1.87; desviación estándar $=0.75$ ); equipamiento $($ media $=1.87$; desviación estándar $=0.75$ ), aprovechamiento del alumno (3 reactivos) (media $=1.78$; desviación estándar $=0.62$ ); asistencia y puntualidad $($ media $=1.73$; desviación estándar $=0.67)$, infraestructura y salario $($ media $=2.33 ;$ desviación estándar $=0.98)$, y desempeño y comunicación $($ media $=1.77$; desviación estándar $=0.62$ ). En conjunto, los valores fueron los siguientes: KMO de 0.846, varianza explicada de 56.63 y alfa de Cronbach de 0.897 (Ver tabla 3).

En la subescala Gestión y actividades directivas se formaron cuatro factores: gestión administrativa $($ media $=2.32 ;$ desviación estándar $=0.98)$, planeación $($ media $=2.24$; desviación estándar $=0.86)$, gestión docente $($ media $=1.95 ;$ desviación estándar $=0.91)$ y relación con padres $($ media $=1.87$; desviación estándar $=0.75)(20$ reactivos en total $)$. En conjunto, los valores fueron los siguientes: KOM de 0.709, varianza explicada de 50.76 y alfa de Cronbach de 0.848 .

Finalmente, en la subescala sobre el alumnado se formaron cuatro factores constituidos por 13 reactivos: mejora del rendimiento de los alumnos (media = 2.18; desviación estándar $=1.18)$, motivación y capacitación $($ media $=1.86$; desviación estándar $=$ 0.98), problemas $($ media $=3.48 ;$ desviación estándar $=1.12)$ y más problemas $($ media $=$ 


\section{Revista Iberoamericana de las Ciencias Sociales y Humanísticas}

2.37; desviación estándar $=1.19$ ). En conjunto, los valores fueron los siguientes: KMO de 0.793, varianza explicada de 61.33 y alfa de Cronbach de 0.762 .

Tabla 3. Subescalas que constituyen a cada factor de la función directiva, número de reactivos que las componen, medias, desviación estándar promedio, pesos factoriales,

KOM, varianza explicada y alfa de Cronbach

\begin{tabular}{|c|c|c|c|c|c|c|c|}
\hline & $\begin{array}{l}\text { Número de } \\
\text { reactivos }\end{array}$ & $\begin{array}{l}\text { Medi } \\
\text { a }\end{array}$ & $\mathrm{DE}$ & $\mathrm{PF}$ & KMO & VE & $\begin{array}{c}\text { a de } \\
\text { Cronbach }\end{array}$ \\
\hline \multicolumn{8}{|c|}{ Trabajo docente } \\
\hline Trabajo en equipo & 6 & 2.69 & 0.99 & $\begin{array}{l}\text { De } 0.42 \mathrm{a} \\
0.76\end{array}$ & \multirow[t]{3}{*}{0.766} & \multirow[t]{3}{*}{47.13} & \multirow[t]{3}{*}{0.71} \\
\hline $\begin{array}{l}\text { Actividades } \\
\text { integradoras }\end{array}$ & 4 & 1.98 & 1.04 & $\begin{array}{c}\text { De } 0.52 \mathrm{a} \\
0.74\end{array}$ & & & \\
\hline $\begin{array}{l}\text { Actividades } \\
\text { mecánicas }\end{array}$ & 3 & 3.09 & 1.11 & $\begin{array}{l}\text { De } 0.36 \text { a } \\
0.76\end{array}$ & & & \\
\hline Materiales NTIC & 4 & 3.61 & 1.19 & $\begin{array}{c}\text { De } 0.56 \mathrm{a} \\
0.86\end{array}$ & \multirow[t]{2}{*}{0.785} & \multirow[t]{2}{*}{61.13} & \multirow[t]{2}{*}{0.77 . } \\
\hline $\begin{array}{l}\text { Materiales } \\
\text { tradicionales }\end{array}$ & 3 & 3.33 & 1.31 & $\begin{array}{c}\text { De } 0.65 \text { a } \\
0.80\end{array}$ & & & \\
\hline Actividades creativas & 5 & 2.80 & 1.20 & $\begin{array}{l}\text { De } 0.48 \mathrm{a} \\
0.75\end{array}$ & \multirow[t]{3}{*}{0.836} & \multirow[t]{3}{*}{47.37} & \multirow[t]{3}{*}{0.80} \\
\hline $\begin{array}{l}\text { Actividades } \\
\text { extrainstitucionales }\end{array}$ & 4 & 3.63 & 1.17 & $\begin{array}{l}\text { De } 0.56 \mathrm{a} \\
0.76\end{array}$ & & & \\
\hline Obligaciones docentes & 5 & 1.44 & 0.70 & $\begin{array}{l}\text { De } 0.45 \text { a } \\
0.83\end{array}$ & & & \\
\hline \multicolumn{8}{|c|}{ Condiciones de trabajo y clima organizacional } \\
\hline $\begin{array}{l}\text { Condiciones } \\
\text { materiales } \\
\text { institucionales }\end{array}$ & 27 & 2.29 & 1.01 & $\begin{array}{c}\text { De } 0.36 \mathrm{a} \\
0.81\end{array}$ & \multirow[t]{3}{*}{0.912} & \multirow[t]{3}{*}{41.40} & \multirow[t]{3}{*}{0.95} \\
\hline $\begin{array}{l}\text { Desempeño de los } \\
\text { docente }\end{array}$ & 18 & 1.74 & 0.71 & $\begin{array}{c}\text { De } 0.39 \mathrm{a} \\
0.72\end{array}$ & & & \\
\hline $\begin{array}{l}\text { Servicios } \\
\text { institucionales }\end{array}$ & 15 & 2.96 & 1.21 & $\begin{array}{c}\text { De } 0.35 \mathrm{a} \\
0.70\end{array}$ & & & \\
\hline \multicolumn{8}{|c|}{ Conocimiento del modelo competencial } \\
\hline $\begin{array}{l}\text { Capacitación y } \\
\text { conocimiento }\end{array}$ & 4 & 2.14 & 0.80 & $\begin{array}{c}\text { De } 0.66 \mathrm{a} \\
0.79 \\
\end{array}$ & 0.757 & 56.25 & 0.73 \\
\hline \multicolumn{8}{|c|}{ Propuestas de mejora para el desempeño } \\
\hline $\begin{array}{l}\text { Esfuerzos } \\
\text { institucionales }\end{array}$ & 7 & 1.68 & 0.89 & $\begin{array}{c}\text { De } 0.49 \mathrm{a} \\
0.78\end{array}$ & 0.851 & 46.16 & 0.80 \\
\hline
\end{tabular}

$\mathrm{DE}=$ desviación estándar; $\mathrm{PF}=$ pesos factoriales; $\mathrm{KMO}=$ Keiser-Meyer-Olkin ; VE = varianza explicada

Fuente: Elaboración propia 
Revista Iberoamericana

de las Ciencias Sociales y

Humanísticas

ISSN: $2395-7972$

\section{Perfil de directivos de alumnos de alto y bajo rendimiento}

En este grupo de directores, solo uno de ellos ha obtenido como grado máximo el de normal superior (con más de 10 años de antigüedad en la escuela) y otro el nivel técnico (entre 1 y 5 años), aunque con mucha experiencia ambos y solamente dedicados a esa labor (tabla 4).

Tabla 4. Perfil de directores del grupo de alumnos de alto rendimiento

\begin{tabular}{|c|c|c|c|}
\hline $\begin{array}{l}\text { Antiguiedad en la } \\
\text { escuela }\end{array}$ & $\begin{array}{l}\text { Antigüedad en la } \\
\text { docencia }\end{array}$ & Último grado obtenido & $\begin{array}{l}\text { Realiza } \\
\text { otra } \\
\text { actividad } \\
\text { además de } \\
\text { la docencia }\end{array}$ \\
\hline \multicolumn{4}{|c|}{$\mathrm{COBACH}$} \\
\hline Entre 1 año y 5 años & $\begin{array}{l}\text { Más de } 10 \text { años a } 20 \\
\text { años }\end{array}$ & Maestría & No \\
\hline Entre 1 año y 5 años & $\begin{array}{l}\text { Más de } 10 \text { años a } 20 \\
\text { años }\end{array}$ & Licenciatura o ingeniería & No \\
\hline Más de 10 años & Más de 20 años & Normal superior & No \\
\hline Más de 10 años & $\begin{array}{l}\text { Más de } 10 \text { años a } 20 \\
\text { años }\end{array}$ & Licenciatura o ingeniería & No \\
\hline \multicolumn{4}{|c|}{ CONALEP } \\
\hline Más de 10 años & $\begin{array}{l}\text { Entre } 1 \text { año y } 10 \\
\text { años }\end{array}$ & $\begin{array}{l}\text { Pasante de licenciatura o } \\
\text { ingeniería }\end{array}$ & No \\
\hline Entre 1 año y 5 años & $\begin{array}{l}\text { Entre } 1 \text { año y } 10 \\
\text { años }\end{array}$ & Técnico & No \\
\hline Más de 10 años & Más de 20 años & Maestría & No \\
\hline \multicolumn{4}{|c|}{ DGETI } \\
\hline Entre 1 año y 5 años & Más de 20 años & Pasante de maestría & No \\
\hline $\begin{array}{l}\text { Más de } 5 \text { años a } 10 \\
\text { años }\end{array}$ & $\begin{array}{l}\text { Entre } 1 \text { año y } 10 \\
\text { años }\end{array}$ & Pasante de maestría & No \\
\hline Entre 1 año y 5 años & $\begin{array}{l}\text { Más de } 10 \text { años a } 20 \\
\text { años }\end{array}$ & Pasante de maestría & No \\
\hline Más de 10 años & Más de 20 años & Licenciatura o ingeniería & No \\
\hline Menos de 1 año & $\begin{array}{l}\text { Más de } 10 \text { años a } 20 \\
\text { años }\end{array}$ & Candidato a doctor & No \\
\hline \multicolumn{4}{|c|}{$\begin{array}{r}\text { Privadas } \\
\end{array}$} \\
\hline Menos de 1 año & $\begin{array}{l}\text { Más de } 10 \text { años a } 20 \\
\text { años }\end{array}$ & Maestría & No \\
\hline Más de 10 años & $\begin{array}{l}\text { Más de } 10 \text { años a } 20 \\
\text { años }\end{array}$ & Licenciatura o ingeniería & Sí \\
\hline Entre 1 año y 5 años & $\begin{array}{l}\text { Más de } 10 \text { años a } 20 \\
\text { años }\end{array}$ & Licenciatura o ingeniería & No \\
\hline
\end{tabular}

Fuente: Elaboración propia 
Revista Iberoamericana

de las Ciencias Sociales y

Humanísticas

ISSN: $2395-7972$

En la tabla 5 aparecen los directores de los planteles con alumnos de bajo rendimiento.

Tabla 5. Perfil de directores del grupo de alumnos de bajo rendimiento

\begin{tabular}{|c|c|c|c|}
\hline $\begin{array}{l}\text { Antigüedad en la } \\
\text { escuela }\end{array}$ & $\begin{array}{l}\text { Antigüedad en la } \\
\text { docencia }\end{array}$ & Último grado obtenido & $\begin{array}{l}\text { Realiza } \\
\text { otra } \\
\text { actividad } \\
\text { además de } \\
\text { la docencia }\end{array}$ \\
\hline \multicolumn{4}{|c|}{$\mathrm{COBACH}$} \\
\hline Más de 10 años & Más de 10 años a 20 años & Maestría & No \\
\hline Más de 10 años & Más de 10 años a 20 años & $\begin{array}{l}\text { Pasante de licenciatura } \\
\text { o ingeniería }\end{array}$ & No \\
\hline \multicolumn{4}{|c|}{ CONALEP } \\
\hline Entre 1 año y 5 años & Más de 10 años a 20 años & $\begin{array}{l}\text { Licenciatura o } \\
\text { ingeniería }\end{array}$ & $\mathrm{Si}$ \\
\hline Entre 1 año y 5 años & Entre 1 año y 10 años & Técnico & No \\
\hline \multicolumn{4}{|c|}{ DGETI } \\
\hline Más de 10 años & Más de 20 años & $\begin{array}{l}\text { Licenciatura o } \\
\text { ingeniería }\end{array}$ & No \\
\hline $\begin{array}{l}\text { Más de } 5 \text { años a } 10 \\
\text { años }\end{array}$ & Más de 10 años a 20 años & Maestría & No \\
\hline Entre 1 año y 5 años & Más de 20 años & Pasante de maestría & No \\
\hline Entre 1 año y 5 años & Entre 1 año y 10 años & $\begin{array}{l}\text { Licenciatura o } \\
\text { ingeniería }\end{array}$ & No \\
\hline Menos de 1 año & Más de 10 años a 20 años & Candidato a doctor & No \\
\hline \multicolumn{4}{|c|}{$\begin{array}{r}\text { Privadas } \\
\end{array}$} \\
\hline Menos de 1 año & Más de 10 años a 20 años & $\begin{array}{l}\text { Licenciatura o } \\
\text { ingeniería }\end{array}$ & No \\
\hline Entre 1 año y 5 años & Más de 10 años a 20 años & $\begin{array}{l}\text { Licenciatura o } \\
\text { ingeniería }\end{array}$ & No \\
\hline Más de 10 años & Más de 20 años & Normal superior & No \\
\hline Entre 1 año y 5 años & Más de 10 años a 20 años & $\begin{array}{l}\text { Licenciatura o } \\
\text { ingeniería }\end{array}$ & $\mathrm{Si}$ \\
\hline
\end{tabular}

Fuente: Elaboración propia

Si se comparan los datos de las tablas 4 y 5 , se observa que prácticamente no hay diferencia en los datos recabados sobre las características de los directores. Por lo tanto, surge una interrogante: ¿por qué en uno y otro caso el rendimiento de los alumnos es distinto? Para tratar de ofrecer una respuesta, revisemos el perfil de los directivos utilizando un modelo de análisis discriminante. 


\section{Análisis discriminante}

Se realizó un análisis multivariado discriminante para evaluar las diferencias entre los directores de estudiantes con alto y bajo rendimiento. Se calculó una función discriminante a partir de las variables contenidas en los cuestionarios, con un nivel de significación de 95 $\%(\mathrm{p} \leq 0.05)$. Este análisis nos permitió responder a dos aspectos fundamentales:

1. Existencia de diferencias entre los grupos de estudiantes con alto y bajo rendimiento.

2. Variables en que se presentaron las diferencias.

En relación con el análisis multivariado clasificador discriminante, se obtuvieron los siguientes resultados:

1. En las funciones canónicas discriminantes se consiguió una sola función con un autovalor superior a 1 (1.221) y una correlación canónica elevada (0.742), lo cual muestra diferencias entre los grupos a partir de las variables incluidas para este análisis (tabla 6).

Tabla 6. Funciones canónicas discriminantes (autovalores)

\begin{tabular}{|c|c|c|c|c|}
\hline \multicolumn{5}{|c|}{ Autovalores } \\
\hline Función & $\begin{array}{c}\text { Autovalo } \\
\mathrm{r}\end{array}$ & $\begin{array}{c}\% \text { de } \\
\text { varianza }\end{array}$ & $\%$ acumulado & $\begin{array}{c}\text { Correlación } \\
\text { canónica }\end{array}$ \\
\hline 1 & 1.221 & 100.0 & 100.0 & 0.742 \\
\hline
\end{tabular}

Fuente: Elaboración propia

2. La prueba de M de Box para igualdad de las covarianzas de las matrices mostró un determinante de 2.469 y su transformación a una $\mathrm{F}$ de 0.379 , lo cual correspondió con una significación de $\mathrm{p}=0.893$. Estos valores permiten aceptar la hipótesis de homogeneidad de covarianzas de las matrices entre los grupos, lo que equivale a decir que las variables involucradas en el análisis se distribuyen de manera similar entre los grupos.

3. En la prueba para determinar la significación de la diferencia entre los grupos a partir de la función calculada, se obtuvo un valor de Lambda de Wilks de 0.450 y un valor de Chi-Cuadrada de 31.528, y $\mathrm{p}=0.000$, por lo que fue significativa la diferencia o discriminación entre los grupos a partir del comportamiento de las variables incluidas en el análisis y evaluadas en esta función discriminante (tabla 7). 
Revista Iberoamericana

de las Ciencias Sociales y

Humanísticas

ISSN: $2395-7972$

Tabla 7. Valores de Lambda de Wilks, Chi-Cuadrada y nivel de significancia

\begin{tabular}{|lc|c|c|c|c|}
\hline \multicolumn{5}{|c|}{ Lambda de Wilks } \\
\hline $\begin{array}{l}\text { Prueba } \\
\text { funciones }\end{array}$ & de & $\begin{array}{c}\text { Lambda de } \\
\text { Wilks }\end{array}$ & Chi-cuadrada & gl & Sig. \\
\hline 1 & 0.450 & 31.528 & 3 & 0.000 \\
\hline
\end{tabular}

Fuente: Elaboración propia

4. El comportamiento de los coeficientes canónicos estandarizados de la función discriminante hallada, así como el análisis de la estructura de la matriz mostraron que las variables con mayor peso en las diferencias entre los grupos fueron juntas y cursos (1.193), infraestructura y salario (-1.278) y problemas (0.484) (tabla 8).

Tabla 8. Coeficientes de función discriminante canónica estandarizados

\begin{tabular}{|l|c|}
\hline & Función \\
\hline & 1 \\
\hline $\begin{array}{l}\text { Juntas } \begin{array}{l}\text { Cursos } \\
\text { y }\end{array} \\
\text { Infra y Salario }\end{array}$ & 1.193 \\
\hline Problemas & -1.278 \\
\hline
\end{tabular}

Fuente: Elaboración propia

5. La función discriminante para estos grupos mostró valores de -1.054 para el grupo centroide de bajo desempeño, y de 1.105 para el alto desempeño, lo que significa una distancia considerable en la representación de estos grupos comparados, a partir del comportamiento de las variables empleadas en la muestra de estudio (tabla 9).

Tabla 9. Funciones en centroides de grupo

\begin{tabular}{|l|c|}
\hline $\begin{array}{l}\text { Logro alto y } \\
\text { bajo }\end{array}$ & 1 \\
\hline Bajo & -1.054 \\
\hline Alto & 1.105 \\
\hline
\end{tabular}

Fuente: Elaboración propia

Las funciones discriminantes canónicas sin estandarizar se han evaluado en medias de grupos.

6. Se consideró buena la capacidad de esta función para pronosticar, pues se logó clasificar correctamente en el grupo de procedencia a 21 de 22 directores del grupo de bajo 


\section{Revista lberoamericana de las Ciencias Sociales y Humanísticas}

ISSN: $2395-7972$

desempeño (95.5\%), y a 17 de 21 directores de alto rendimiento ( $81 \%)$. Finalmente, de manera general, se obtuvo $88.4 \%$ de directores de la muestra clasificados correctamente (tabla 10).

Tabla 10. Resultados de clasificación

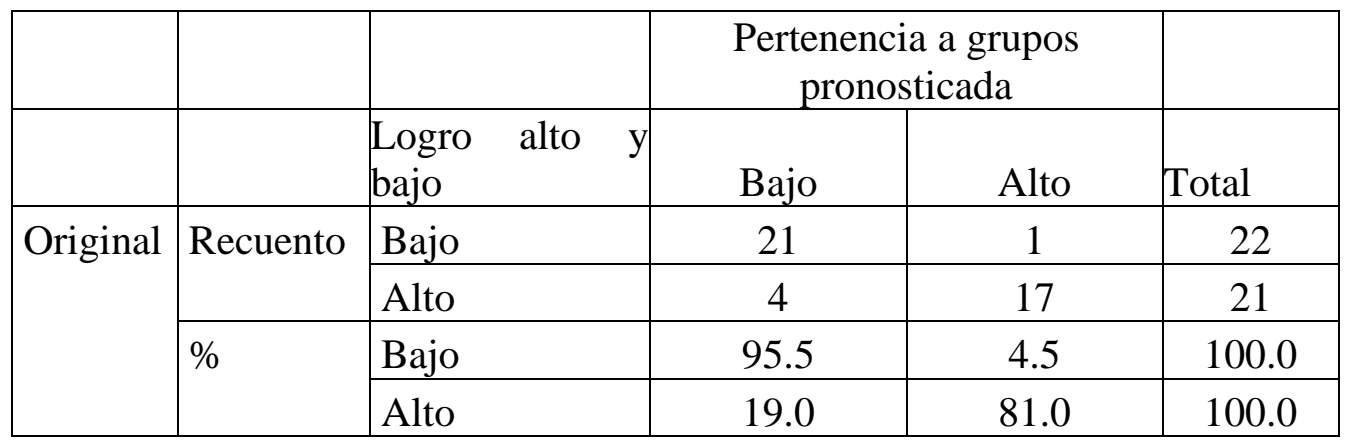

Fuente: Elaboración propia

De manera general, las conclusiones principales derivadas de los resultados del análisis discriminante fueron las siguientes:

1. La función discriminante calculada permitió diferenciar a los directores de los grupos de alto y bajo desempeño, con una efectividad de pronóstico de $88.40 \%$ (p $\leq 0.005)$ en esta muestra de estudio.

2. Los variables con mayor contribución a las diferencias entre ambos grupos fueron juntas y cursos, infraestructura y salario y problemas.

3. Si consideramos el valor de la correlación canónica (0.742), el valor de la prueba de $\mathrm{M}$ de Box (2.469 y su transformación a una $\mathrm{F}$ de 0.379 , con una $\mathrm{p}=0.893$ ), el valor de la Lambda de Wilks (0.450), el valor de Chi-Cuadrada (31.528, y p = 0.000) y la buena capacidad de esta función para pronosticar correctamente (95.5 $\%$ en los directores del grupo de bajo desempeño, $81 \%$ en los de alto rendimiento y $88.4 \%$ de manera general), el análisis discriminante, de facto, se convierte en un ejercicio de validez convergente para el instrumento de contexto de directores.

\section{Discusión}

La escuela, como espacio de formación intermedio entre la familia y la comunidad, refuerza, reproduce pero también transforma los procesos sociales, por lo que el desarrollo de habilidades sociales en sus estudiantes es indispensable para convivir en un escenario diverso y cambiante (Gómez, Del Rey, Romera y Ortega, 2015). 


\section{Revista Iberoamericana de las Ciencias Sociales y Humanísticas}

El problema radica, sin embargo, en que el desarrollo de esas destrezas suele estar condicionado por múltiples variables, como la edad, el sexo, el turno cursado, el semestre, etc. (Chain, Cruz, Martínez y Jacomé, 2003; Moreira, 2006). De hecho, y aunque a veces no se toma en cuenta, la figura del director también resulta importante en ese propósito porque sus acciones tienen un efecto directo sobre las estructuras, los procesos y los demás miembros de la escuela (Hallinger, 2003; Harris, 2009; Rojas, 2006). En efecto, para asegurar un ambiente adecuado de aprendizaje es necesario que el director ejerza un liderazgo efectivo que promueva las condiciones para un sano desarrollo de los estudiantes (Sánchez, 2011; Maureira, 2004). El liderazgo es un componente de la gestión directiva que comprende la capacidad de orientar y motivar a los subordinados para el cumplimiento de las actividades y los objetivos escolares en forma conjunta (Cayulef, 2007; García, 2010; Murillo, 2006; Ortega y Rocha, 2011).

Además, el director de una institución educativa es la pieza central en el esquema general de gobierno y gobernabilidad. Por ello, para responder a las problemáticas académicas, burocráticas y políticas debe contar con las habilidades del gerente, del burócrata y del príncipe, "mezclando así las habilidades políticas del príncipe con las exigencias administrativas del burócrata y las actitudes gerenciales asociadas a la gestión" (Acosta, 2009, p. 11).

Lo anterior debe llevarnos a considerar que el director es (o debería ser) un factor determinante para influir en el mejoramiento de estudiantes con bajo rendimiento. Esto lo puede conseguir gracias a su perfil pedagógico, sus posibilidades de comunicación efectiva y sus habilidades interpersonales para reunirse con los padres de familia y con los profesores en general. Además, debe tener la capacidad para gestionar la adquisición de nuevo equipo, así como para impulsar cursos de capacitación para maestros, proyectos de investigación, actividades culturales, deportivas y sociales, etc.

Por eso, Leithwood (1994, citado por Vázquez, 2013) señala que los efectos del liderazgo de los directores explican (directa e indirectamente) $25 \%$ de los logros escolares. De hecho, según el mismo autor, el liderazgo escolar sería la segunda variable más importante en el aprendizaje de los alumnos, lo cual de alguna forma se ve reflejado en la presente investigación. Es decir, hemos encontrado que las variables con mayor contribución a las diferencias entre los grupos de alto y bajo rendimiento fueron la organización de juntas de trabajo, los cursos de capacitación docente, la preocupación por el estado que guarda la 
infraestructura del plantel, así como por el salario. Esto coincide con los hallazgos de Bravo y Verdugo (2007) y Murillo y Krichesky (2012), quienes señalan que los directores son percibidos por los docentes como grandes e influyentes líderes.

Ahora bien, dentro del análisis discriminante entre directores, las variables con mayor contribución a las diferencias entre los grupos de alto y bajo desempeño fueron las de organizar juntas y cursos (con y para los profesores), dedicar tiempo y esfuerzo para mejorar las condiciones de la infraestructura (fuera de las posibilidades del presupuesto asignado), así como la atención pronta, efectiva y eficaz de los problemas que cotidianamente se presentan en los planteles. Esto nos demuestra que las acciones que quedan en su radio de acción pueden coadyuvar a la generación de estudiantes de alto rendimiento. Todo lo demás (es decir, las decisiones y acciones que quedan definidas por las administraciones centrales) en nada contribuyen a lo anterior. Por eso, resultaría interesante dotar de cierto nivel de autonomía a los directores para decidir lo relevante en los aprendizajes, pues de ese modo se podría estimar hasta dónde esas acciones contribuyen con la mejoría del rendimiento estudiantil.

\section{Conclusiones}

Se concluye que el director es el actor escolar con mayor poder para mejorar los logros académicos de los alumnos de educación media, aunque cabe acotar que su desempeño está altamente influenciado por las características de su equipo escolar y especialmente por sus docentes. En efecto, el director configura algunas variables escolares que afectan la respuesta del docente ante la búsqueda de opciones didácticas y materiales educativos. Por eso, debe integrar de forma colectiva a todo su equipo en las actividades para el desarrollo de competencias académicas. La percepción de capacidad como colectivo es una variable altamente influyente en la actuación docente, por lo que el director debe poner especial empeño en mejorar la confianza de los docentes como equipo para alcanzar los objetivos académicos.

Los resultados, en definitiva, indican que los directores de educación media superior deben tomar un papel más activo, ya que sin su apoyo y guía, los docentes difícilmente podrán estructurar entornos escolares que permitan la formación de individuos capaces de mostrar competencias académicas básicas de matemáticas, español y ciencias, así como las requeridas para convivir y participar en el mejoramiento de una sociedad democrática. 


\section{Futuras líneas de investigación y limitaciones}

Una línea de investigación futura se debería enfocar en el uso de una metodología mixta. Por ejemplo, si se desea estudiar el fenómeno del logro académico desde una perspectiva escolar, es importante escuchar las voces de otros informantes, como docentes, alumnos, etc. Por otra parte, se recomienda continuar estudiando la relación entre liderazgo y apoyo directivo, y su impacto sobre el logro académico, pues se ha percibido que solamente las acciones que quedan en su radio de acción son las que efectivamente coadyuvan a la generación de estudiantes con alto desempeño

Asimismo, se recomienda una investigación de corte longitudinal para conocer el sentido de las relaciones. Igualmente, se sugiere la reproducción de este modelo a partir de un análisis multinivel donde se distingan las variables colectivas de las individuales, pues eso permitiría identificar cuál tipo de variable tiene mayor impacto sobre las competencias académicas y de comportamiento social (Morgeson y Hofman, 1999). Es necesario considerar que la medición de variables que indaguen sobre la relación director-subordinado funcionarán mejor a nivel personal que global, ya que estarán influenciadas por la experiencia personal de cada individuo con el director (Choi et al., 2003).

En cuanto a las limitaciones de este trabajo, se pueden mencionar aquellas relacionadas con el uso del autorreporte, el cual es el más utilizado en evaluaciones escolares y resulta con una alta deseabilidad social (Lillehoj, Griffin y Spoth, 2004). Otra limitación fue que solo trabajó con los directores, por lo que se deben incluir en futuras indagaciones a alumnos y docentes (Bauman y Del Río, 2005).

Finalmente, se debe subrayar que son pocos los estudios que abordaran el fenómeno del logro escolar de los alumnos desde la perspectiva de los directivos. Por último, el corte transversal empleado no permitió establecer el orden de causalidad entre las variables, por lo que fue limitada la comprensión del comportamiento de las variables escolares incluidas en el presente estudio. 


\section{Referencias}

Acosta, A. (2009). Príncipes, burócratas y gerentes. El gobierno de las universidades públicas en México. México: ANUIES.

Bauman, S. and Del Río, A. (2005). Knowledge and beliefs about bullying in schools: Comparing pre-service teachers in the Unites States and the United Kingdom. School Psychology International, 26(4), 428-442.

Bitar, S. (2012). Cómo construir consensos en educación. Promoción de la Reforma Educativa en América Latina y el Caribe. Recuperado de http://www.thedialogue.org/wp-content/uploads/2012/11/preal-63-bitar-comoconstruir-consensos-en-educacic3b3n.pdf

Bravo, M. y Verdugo S. (2007). Gestión escolar y éxito académico en condiciones de pobreza. Revista Electrónica Iberoamericana sobre Calidad, Eficacia y Cambio en Educación, 5(1), 121-44. Recuperado de https://www.redalyc.org/articulo.oa? $\mathrm{id}=55100107$

Bustamante, Y., Székely, M. y Martínez, M. (2017). Decisiones sobre la educación media superior en México. Gaceta de la Política Nacional de Evaluación Educativa en México, 3(7), 70-74.

Cayulef, C. (2007). El liderazgo distribuido una apuesta de dirección escolar de calidad. Revista Iberoamericana sobre Calidad, Eficacia y Cambio en Educación, 5(5), 144148.

Chain, R. (1995). Estudiantes universitarios: trayectorias escolares. Universidad Veracruzana.

Chain, R., Cruz, N., Martínez, N. y Jacomé, N. (2003). Examen de selección y probabilidad de éxito escolar en estudios superiores. Estudio en una universidad pública estatal mexicana. REDIE. Revista Electrónica de Investigación Educativa, 5(1).

Choi, I., Dalal, R., Kim-Prieto, C. and Park, H. (2003). Culture and judgement of causal relevance. Journal of Personality and Social Psychology, 84(1), 46-59. Doi: https://doi.org/10.1037/0022-3514.84.1.46

De la Cruz, I. (2016). Elegir un bachillerato: el prestigio como factor determinante para los alumnos y sus familias. Innovación Educativa, 16(70), 111-130. Recuperado de http://www.scielo.org.mx/pdf/ie/v16n70/1665-2673-ie-16-70-00111.pdf 
Revista Iberoamericana

de las Ciencias Sociales y

Humanísticas

ISSN: $2395-7972$

Elmore, R. F. (2004). School reform from the inside out: Policy, practice, and performance. Harvard University Press.

Fierros, L. (2014) Estimación de la relación entre variables de contexto y de desempeño en Educación Media Superior del estado de Sonora (tesis de doctorado). Universidad del Valle de México, Hermosillo, Sonora.

Garay, S. y Uribe, M. (2006). Dirección escolar como factor de eficacia y cambio situación de la dirección escolar en Chile. Revista Electrónica Iberoamericana sobre Calidad, Eficacia y Cambio en Educación, 4(4), 39-64. Recuperado de https://www.redalyc.org/pdf/551/55140405.pdf

García, I. (2010). Liderazgo distribuido, una visión innovadora de la dirección escolar: una perspectiva teórica. Omnia, 16(3), 19-36.

Gómez, O., Del Rey, R., Romera, E. y Ortega, R. (2015). Los estilos educativos paternos y maternos en la adolescencia y su relación con la resiliencia, apego y la implicación en acoso escolar. Anales de Psicología, 31(3), 979-989. Doi: https://doi.org/10.6018/analesps.31.3.180791

Guzmán, F. (2018). La experiencia de la evaluación docente en México: análisis crítico de la imposición del servicio profesional docente. Revista Iberoamericana de Evaluación Educativa, 11(1), 135-158. Doi: https://doi.org/10.15366/riee2018.11.1.008

Hallinger, P. (2003). Leading Educational Change: reflections on the practice of instructional and transformational leadership. Journal of Education, 33, 329-352.

Harris, A. (2009). Distributed leadership: What we know. In Harris, A. (ed.), Distributed Leadership (pp. 11-21). Springer.

Horbath, J. y Gracia, M. (2014). La evaluación educativa en México. Revista de Relaciones Internacionales, Estrategia y Seguridad, 9(1), 59-85. Recuperado de www.redalyc.org/articulo.oa?id=92731211003

Hunt, B. (2009). Efectividad del desempeño docente. Una reseña de la literatura internacional y su relevancia para mejorar la educación en América Latina. Programa de Promoción de la Reforma Educativa en América Latina y el Caribe.

Lillehoj, C., Griffin, K. and Spoth, R. (2004). Program provider and observer rat- ings of school-based preventive intervention implementation: Agreement and relation to youth outcomes. Health Education \& Behavior, 31, 242-57. Doi: doi:10.1177/1090198103260514 
Revista Iberoamericana

de las Ciencias Sociales y

Humanísticas

ISSN: $2395-7972$

López, P. (2010). Variables asociadas a la gestión escolar como factor de calidad educativa. Estudios Pedagógicos, 36(1), 147-158. Doi: http://dx.doi.org/10.4067/S071807052010000100008

Maureira, O. (2004). El liderazgo factor de eficacia escolar, hacia un modelo causal. Revista Electrónica Iberoamericana sobre Calidad, Eficacia y Cambio en Educación, 2(1). Recuperado de_https://www.redalyc.org/pdf/551/55120108.pdf

Maureira, O. (2006). Dirección y eficacia escolar, una relación fundamental. Revista Electrónica Iberoamericana sobre Calidad, Eficacia y Cambio en Educación, 4(4), 1-10. Recuperado de_https://www.redalyc.org/articulo.oa?id=55140402

Moreira, T. (2006). Estimación de la validez predictiva de las pruebas de bachillerato en educación media. Actualidades en Psicología, 20, 121-140.

Morgeson, F. and Hofmann, D. (1999). The structure and function of collective constructs: Implications for multilevel research and theory development. Academy of Management Review, 24, 249-65.

Murillo, J. (2006). Una dirección escolar para el cambio: del liderazgo transformacional al liderazgo distribuido. Revista Electrónica Iberoamericana sobre Calidad, Eficacia y Cambio en Educación, 4(4), 11-24. Recuperado de https://www.redalyc.org/pdf/551/55140403.pdf

Murillo, J. y Krichesky, G. (2012). El proceso del cambio escolar. Una guía para impulsar y sostener la mejora de las escuelas. Revista Iberoamericana sobre Calidad, Eficacia y Cambio en Educación, 10(1), 26-43. Recuperado de http://www.rinace.net/reice/numeros/arts/vol10num1/art2.pdf

Organización para la Cooperación y el Desarrollo Económicos (OCDE) (2020). Panorama de la educación 2020. Recuperado de https://gpseducation.oecd.org/Content/EAGCountryNotes/EAG2020_CN_MEX_es. pdf

Ortega, M. y Rocha, M. (2011). El ejercicio del liderazgo distribuido desde la función que desempeñan los docentes de educación básica. XI Congreso Nacional de Investigación Educativa, Ciudad de México. Recuperado de http://www.comie.org.mx/congreso/memoriaelectronica/v11/docs/area_13/1422.pdf

Pacheco, R. J., Robles, C. A. y Ospino, A. J. (2018). Análisis de la gestión administrativa en las instituciones educativas de los niveles de básica y media en las zonas rurales de 
Revista Iberoamericana

de las Ciencias Sociales y

Humanísticas

ISSN: 2395 - 7972

Santa Marta, Colombia. Información Tecnológica, 29(5), 259-266. Doi: http://dx.doi.org/10.4067/S0718-07642018000500259

Peniche, R. y Cisneros-Cohernourm, E. (2013). Competencias profesionales de administradores escolares desde la perspectiva de los docentes. En Navarro, M. y Barraza, A. (coords.), Dirección, liderazgo, modelos y procesos de gestión: claves hacia la transformación (pp. 71-104). Red Durango de Investigadores Educativos A. C.

Pérez-Franco, L. (1997). Caracterización y seguimiento de estudiantes de la licenciatura en Sociología, generación 1995 y 1996 (serie 2, nro. 289). UAM-A; DCS; H. México.

Quintana Torres, Y. (2018). Calidad educativa y gestión escolar: una relación dinámica. Educación y Educadores, 21(2), 259-281. Doi: 10.5294/edu.2018.21.2.5

Rojas, A. (2006). La cara oculta de la luna: liderazgo y crítica al racionalismo en la formulación e implementación de políticas educacionales. Revista Electrónica Iberoamericana sobre Calidad, Eficacia y Cambio en Educación, 4(4).

Sánchez, F. (2011). Desafíos de la gestión escolar de los directores de educación preescolar y su proceso de formación (tesis de maestría). Escuela superior de Comercio y Administración, Instituto Politécnico Nacional.

Sardon, D. (2017). Liderazgo transformacional y gestión escolar en instituciones educativas. Revista de Investigación Altoandin, 19(3), 295-304. Doi: http://dx.doi.org/10.18271/ria.2017.294

Secretaría de Educación Pública (SEP) (2019). Nuevo currículo de la educación media superior 2018-2019. Recuperado de http:// http://www.sems.gob.mx/work/models/sems/Resource/12614/1/images/Presentacio n_General.pdf

Sierra-Bravo, R. (1985). Técnicas de investigación social. Teoría y ejercicios. Paraninfo. Tagliacarne, G. (1968). Técnicas y práctica de las investigaciones de mercado. Ariel.

Tolozano, M. R., Ferrer, M. y Forgas, J. (2017). Proyecto de gestión educativa institucional para la formación de técnicos de salud basado en competencias. MEDISAN, 21(4), 470-478.

Uribe, C., López-Córdova E., Mancera, C. y Barrios, M. (2012). México: retos para el sistema educativo 2012-2018. Recuperado de_https://institutomexico.org.mx/wpcontent/uploads/2018/10/Desafios-de-la-educaci\%C3\%B3n-en-M\%C3\%A9xico.pdf 
Revista Iberoamericana

de las Ciencias Sociales y Humanísticas

ISSN: 2395 - 7972

Vázquez, A. (2013). Interdependencia entre el liderazgo transformacional, cultura organizacional y cambio educativo: una reflexión. Revista Iberoamericana sobre Calidad, Eficacia y Cambio en Educación, 11(1), 73-91. Recuperado de http://www.rinace.net/reice/numeros/arts/vol11num1/art5.pdf

Vélez, C. (2012). La gestión de la educación en Colombia 2002-2010. Programa de Promoción de la Reforma Educativa en América Latina y el Caribe.

Vera, J., Fierros, E. y Peña, M. (2014). Medida de gestión directiva para educación media superior. En Jaik, A. y Málaga. S. (coord.), Las competencias y su relación con ... La gestión, la investigación, la docencia, el desarrollo profesional (pp. 72-91). Red Durango de Investigadores Educativos, A.C. (Ed).

Vera, J., Huesca, L., y Laborín, J. (2011). Logro y tasas de riesgo en alumnos de alto y bajo desempeño escolar en el nivel medio superior en Sonora. Perfiles Educativos, $33(132)$, 48-66. Recuperado

de http://www.scielo.org.mx/pdf/peredu/v33n132/v33n132a4.pdf 


\section{Revista Iberoamericana de las Ciencias Sociales y Humanísticas}

\begin{tabular}{|c|c|}
\hline Rol de Contribución & Autor (es) \\
\hline Conceptualización & $\begin{array}{l}\text { Jose ángel vera noriega (igual) } \\
\text { Luis Enrique Fierros Dávila (igual) }\end{array}$ \\
\hline Metodología & $\begin{array}{l}\text { Jose ángel vera noriega (igual) } \\
\text { Luis Enrique Fierros Dávila (igual) }\end{array}$ \\
\hline Software & No aplica \\
\hline Validación & $\begin{array}{l}\text { Jose ángel vera noriega (igual) } \\
\text { Luis Enrique Fierros Dávila (igual) }\end{array}$ \\
\hline Análisis Formal & $\begin{array}{l}\text { Jose ángel vera noriega (igual) } \\
\text { Luis Enrique Fierros Dávila (igual) }\end{array}$ \\
\hline Investigación & $\begin{array}{l}\text { Jose ángel vera noriega (igual) } \\
\text { Luis Enrique Fierros Dávila (igual) }\end{array}$ \\
\hline Recursos & $\begin{array}{l}\text { Jose ángel vera noriega (igual) } \\
\text { Luis Enrique Fierros Dávila (igual) }\end{array}$ \\
\hline Curación de datos & $\begin{array}{l}\text { Jose ángel vera noriega (igual) } \\
\text { Luis Enrique Fierros Dávila (igual) }\end{array}$ \\
\hline $\begin{array}{l}\text { Escritura - Preparación del } \\
\text { borrador original }\end{array}$ & $\begin{array}{l}\text { Jose ángel vera noriega (igual) } \\
\text { Luis Enrique Fierros Dávila (igual) } \\
\text { Jose Alberto Fraijo Figueroa (igual) }\end{array}$ \\
\hline Escritura - Revisión y edición & $\begin{array}{l}\text { Jose ángel vera noriega (igual) } \\
\text { Luis Enrique Fierros Dávila (igual) } \\
\text { Jose Alberto Fraijo Figueroa (igual) }\end{array}$ \\
\hline Visualización & $\begin{array}{l}\text { Jose ángel vera noriega (igual) } \\
\text { Luis Enrique Fierros Dávila (igual) } \\
\text { Jose Alberto Fraijo Figueroa (igual) }\end{array}$ \\
\hline Supervisión & $\begin{array}{l}\text { Jose ángel vera noriega (igual) } \\
\text { Luis Enrique Fierros Dávila (igual) } \\
\text { Jose Alberto Fraijo Figueroa (igual) }\end{array}$ \\
\hline Administración de Proyectos & $\begin{array}{l}\text { Jose ángel vera noriega (igual) } \\
\text { Luis Enrique Fierros Dávila (igual) } \\
\text { Jose Alberto Fraijo Figueroa (igual) }\end{array}$ \\
\hline Adquisición de fondos & Jose Angel vera Noriega \\
\hline
\end{tabular}

